\title{
ESSENTIALLY POSITIVE SYSTEMS OF LINEAR DIFFERENTIAL EQUATIONS
}

\author{
BY GARRETT BIRKHOFF AND LEON KOTIN
}

Communicated by F. Browder, May 17, 1965

Consider an essentially positive [2] system of linear DE's, that is, any system of the form

$$
d x_{i} / d t=\sum q_{i j}(t) x_{j}, \quad q_{i j}(t)>0 \text { if } i \neq j .
$$

This maps the positive hyperoctant $C$ of real $\left(x_{1}, \cdots, x_{n}\right)$-space into itself. The effect of (1) in a small increment of time $d t$ is, using formulas of Ostrowski [3], to map $C$ into an interior polyhedral cone whose projective diameter is given for $P=\exp [Q(t) d t]=I+Q(t) d t+\cdots$ by

$$
\ln \left\{\sup _{i, j, k, l}\left(p_{k i} p_{l j} / p_{k j} p_{l i}\right)\right\} \text {. }
$$

This is maximized asymptotically (as $d t \downarrow 0$ ) by setting $k=i, l=j$, so that the numerator approaches 1 . Hence the projective diameter of $e^{Q(t) d t}(C)$ is, asymptotically,

$$
\Delta=-\ln \left\{\inf _{i \neq j}\left[\left(q_{i j} q_{j i}\right) d t^{2}\right]\right\}, \quad q_{i j}=q_{i j}(t) .
$$

By a basic theorem of [1], all projective distances in $C$ are therefore contracted by a factor at most

$$
\begin{gathered}
\tanh (\Delta / 4)=\left(1-e^{-\Delta / 2}\right) /\left(1+e^{-\Delta / 2}\right)=1-\psi(t) d t \\
\text { where } \psi(t)=2\left[\inf _{i \neq j} q_{i j}(t) q_{j i}(t)\right]^{1 / 2} .
\end{gathered}
$$

This proves the following basic result.

Lemma. For any essentially positive system (1) of linear DE's, all projective distances in $C$ are contracted by an asymptotic factor at most $1-\psi(t) d t$ in the time interval $(t, t+d t)$, where $\psi(t)$ is given by (4).

Integrating with respect to $t$, we deduce the

ThEOREM. For any essentially positive system (1) of linear DE's, let $\theta(\mathbf{x}(t), y(t))$ denote the projective distance in $C$ between two solutions of (1) which are positive on $[0, \infty)$. Then

$$
\text { (5) } \theta(x(t), y(t)) \leqq \theta(x(0), y(0)) \exp \left[-\int_{0}^{t} \psi(s) d s\right], \quad t>0 .
$$

For example, consider the interesting case $d^{2} x / d t^{2}=p(t) x, p(t)>0$. Then 


$$
Q(t)=\left(\begin{array}{cc}
0 & 1 \\
p(t) & 0
\end{array}\right)
$$

has only one pair of off-diagonal entries, and $\psi(t)=2 \sqrt{ }(p(t))$. Hence we have the

Corollary. Let $x(t)$ and $y(t)$ be any two solutions of $d^{2} x / d t^{2}=p(t) x$ with $p(t)>0, x(0)>0, x^{\prime}(0)>0, y(0)>0, y^{\prime}(0)>0$. Then

$$
\left|\ln \left[\frac{x(t) y^{\prime}(t)}{x^{\prime}(t) y(t)}\right]\right| \leqq\left|\ln \left[\frac{x(0) y^{\prime}(0)}{x^{\prime}(0) y(0)}\right]\right| \exp \left[-2 \int_{0}^{t}(p(s)) d s\right] .
$$

The preceding result is helpful for proving the convergence of all positive solutions of an essentially positive system (1) to a unique limiting asymptotic behavior as $t \rightarrow \infty$.

To treat similarly $d^{3} x / d t^{3}=p(t) x$ with $p(t)>0$, however, the preceding technique must be modified.

\section{REFERENCES}

1. G. Birkhoff, Extensions of Jentzsch's Theorem, Trans. Amer. Math. Soc. 85 (1957), 219-227.

2. G. Birkhoff and R. S. Varga, Reactor criticality and nonnegative matrices, J. SIAM 6 (1958), 354-277.

3. A. Ostrowski, in Recent advances in matrix theory, Univ. of Wisconsin Press, Madison, Wis., 1964.

HARVARD UNIVERSITY AND

U. S. Army Electronics laboratories, Fort Monmouth, New Jersey 